\title{
The locus of the word-priority effect in a target-detection task
}

\author{
JOHN A. SLOBODA \\ University of Keele, Keele, Staffordshire, England ST5 $5 B G$
}

\begin{abstract}
When subjects are required to monitor verbal displays for predesignated targets, their reaction time for word targets is less than that for subcomponents of words, such as letters. One explanation for this word-priority effect is that encoding of words is completed prior to encoding of their constituent letters. An alternative explanation is that it takes longer to compare letter targets with encoded displays than it does word targets. In this experiment, target set size and display illumination were independently manipulated during word and letter target-detection tasks, and interactions between factors were examined using the assumptions of Sternberg's (1969) additive factor approach. It was found that the magnitude of the word-priority effect increased with an increase in target set size, but not with a decrease in display illumination. This suggested that the effect occurs at a comparison stage rather than at an encoding stage in the information processing chain.
\end{abstract}

There now exists considerable evidence for a phenonemon which may be called the word-priority effect. It has similar paradoxicality to the much studied word-superiority effect (Reicher, 1969; Wheeler, 1970). The phenonemon occurs when observers are required to detect the occurrence of linguistic targets such as words, syllables, letters, or phonemes in visually or auditorily presented verbal material. A number of independent studies have confirmed that it takes longer to detect the presence of subcomponents of words, such as phonemes or letters, than it takes to detect the presence of words themselves (Ball, Wood, \& Smith, 1975; Foss \& Swinney, 1973; Johnson, 1975: McNeil \& Lindig, 1973; Savin \& Bever, 1970; Sloboda, 1976).

The word-priority effect (WPE) differs from the word-superiority effect (WSE) in two ways. In the WPE, stimuli are presented under fairly normal conditions of exposure, whereas, in the WSE, signal-to-noise ratio is lowered (usually by very brief exposure durations). Secondly, in the WPE, the stimulus type remains constant across conditions (a word or a group of words) and the effect is demonstrated by varying target type (e.g., letter vs word), whereas, in the WSE, the target type remains constant across conditions (usually a single letter) and the effect is demonstrated by varying stimulus type (e.g., letter vs word). These differences make it unclear that the two effects necessarily have identical causes, despite their surface similarities. The present study, therefore, treats the WPE as a totally independent phenonemon and leaves for the future the development of a model which might account for both effects.

The author would like to thank G. Davies for help with the design of the filter used in the experiment, and A. Burgess-Webb for assistance during the experimental sessions.
An example of the WPE is given by Johnson (1975), who asked subjects to judge whether a visually presented word contained a predesignated target letter (letter matching), and compared this with a condition in which he asked them to judge whether a visually presented word was a predesignated target word (word matching). Johnson found that the lettermatching task took significantly longer than the wordmatching task, and argued from this (similarly to Foss \& Swinney, 1973) that words must be recognized prior to their component letters. However, Sloboda (1976) has argued (in common with Ball et al., 1975, and McNeil \& Lindig, 1973) that the effect is not necessarily a result of encoding or recognition stages in the task, but may reflect differences at a stage of comparison between the encoded stimulus and the memory target. For instance, a target letter must be compared with several stimulus letters, whereas a target word may be compared only with a single name code for the whole word. If comparisons occur serially, then the observed reaction time (RT) relationships are to be predicted, since letter matching requires more comparisons than does word matching.

It must be emphasized, however, that a serial model is not the only type that could account for the WPE in terms of a comparison process. A parallel model in which individual comparison times were directly related to the number of comparisons to be made would account for such data; among serial models, a left-to-right scan or a random-access process would be equally acceptable. It is not the concern of the present study to decide between these models: rather, the aim is to establish whether any such model should be invoked to account for the WPE. If the effect turns out to be an encoding effect, as Johnson (1975) has argued, then this whole class of comparison models is ruled out. 
Sternberg (1969) has developed a method for locating the processing stage at which a factor has its effect on the total RT in an information processing task. A central assumption of this additive factor method is that factors affecting the same processing stage will interact, whereas variables affecting only different stages will not interact. Following Sternberg (1969) and others (e.g., Massaro, 1975), one may suppose that the typical task in which a word-priority effect is found contains (among others) an encoding stage, whose duration will be susceptible to factors influencing stimulus quality (such as luminance), and a comparison stage, whose duration will be determined by the number and nature of the comparisons to be made (such as size of positive memory set).

Given these assumptions, then, if the word-priority effect occurs because words are encoded prior to letters, the effect should interact with changes in stimulus luminance. Johnson's (1975) model for the matching task supposes that letter recognition requires additional processing of stimulus information to that required for word encoding. Decreasing stimulus luminance by a given amount should, on this account, increase both word and letter encoding time by a proportional constant, thus increasing the arithmetic RT difference between the two tasks. If, on the other hand, the word-priority effect occurs because the comparison between a target word and a stimulus can take place faster than the comparison between a target letter and a stimulus, then the effect should interact with changes in the number of positive targets. Increasing target set size should, on this account, increase both word and letter comparison time by a proportional constant, thus increasing the arithmetic RT difference between the two tasks.

The experiment to be reported here examined the effect on RT in a target-detection task of three principal factors: stimulus luminance, target set size, and nature of target (word or letter). It did this using a within-subjects factorial design that allowed examination of all main effects and interactions due to these three factors. A fourth factor, orthogonal to the preceding three, was contributed by the design feature that the target was present in the stimulus on only half of the trials.

\section{METHOD}

Displays were presented in a Cambridge two-field tachistoscope. Each display remained in view until the subject responded. The rate of presentation of displays was about one every $10 \mathrm{sec}$. Each subject had by his side a checklist of target items numbered in sequence, one for each display. Prior to each display, the subject read the appropriate target item(s). He indicated his response by pressing one of two thumbswitches. The experimenter recorded both the response and the RT from display onset.

\section{Materials and Design}

A deck of 48 cards was prepared, each card bearing a common four-letter word, typed in uppercase, in the center
Table 1

Summary of Experimental Conditions

\begin{tabular}{ll}
\hline \multicolumn{1}{c}{ Condition } & $\begin{array}{c}\text { Example of } \\
\text { Target for Stimulus } \\
\text { Display CAST }\end{array}$ \\
\hline 1a Single-word match & CAST \\
1b Single-word mismatch & CASH \\
2a Double-word match & ROPE CAST \\
2b Double-word mismatch & ROPE CASH \\
3a Single-letter match & T \\
3b Single-letter mismatch & H T \\
4a Double-letter match & E T \\
4b Double-letter mismatch & E H \\
\hline
\end{tabular}

of a white field. The deck was identical to that used in Experiment 3 of a previous study (Sloboda, 1976). Each word was chosen so that it could be paired with a target word which differed from it by only one letter. A quarter of the word pairs so formed differed at each letter position within the word. Each subject had two separate runs through the deck, once with word targets, and once with letter targets. Targets were presented in the same typeface as the display words. The experimental design required eight different conditions of target-display conjunction. These conditions are summarized in Table 1 . Double targets were presented side by side on the target sheet, and subjects were required to respond "yes" if either target matched the display. Of the two word targets, one was either identical to the display word or differed from it by one letter; the other had letters in common with neither the display nor the other target word. The left-right ordering of the two types of targets was randomly varied on the target sheet. Of the two letter targets, either one of them was contained in the display word or neither of them was.

The example illustrates a control which operated in the letter mismatch conditions. When a mismatch occurred, the target (or one of the two targets) was the letter which, if substituted in the appropriate position in the display word, would form the word used in the word mismatch trial for that particular word. The control operated within subjects, so that, for instance, a subject who saw the word "CAST" with the target "CASH" would see it again with the target "T." Thus, the critical position for comparison in each case was the same: the final letter. Although a single display word was used in Table 1 for illustration, each subject saw a given word only twice, once with a word as target, and once with the appropriately controlled letter as target.

Two complementary target lists were prepared such that word displays which required a mismatch response on one list required a matching response on the other list, and vice versa. Equal numbers of subjects were assigned to each target list. Conditions 1-4 were presented in discrete blocks of 24 presentations each, but the order of presentation was balanced across subjects, such that half the subjects received word targets before letter targets and, within target type, half the subjects received the single-target trials before the double-target trials. Matches and mismatches were distributed within conditions, such that equal numbers of matches and mismatches occurred in a single condition and a given response was correct no more than three times in succession.

Finally, each of the four conditions was split into two blocks of 12 trials. Two levels of luminance for the displays were chosen, and a block was assigned to each level. Half the subjects received a "low luminance" for the first block within each condition. The other half received a "high luminance" first. The total design thus required multiples of 16 subjects to completely balance order of presentation of conditions across both target lists. Luminance was lowered by inserting an opaque filter in front of the stimulus while the subject was viewing the 
fixation field. In this way, the necessity to alter the setting on the exposure lamps of the tachistoscope during the experiment was eliminated. The filter was made by partially exposing finegrain black-and-white photographic film to a uniformly illuminated gray surface, and using the developed negative itself in a filter holder which held the negative flush against the display card. The opacity of the filter was chosen empirically by varying the amount of exposure given to the film and finding a negative which significantly increased RT without introducing phenomenal uncertainty about the identity of the stimulus. The design entailed that the illumination be switched every 12 trials. Within each block of 12 trials, there were six matches and six mismatches.

\section{Procedure}

Each subject was familiarized with the task by five practice trials at each of the double-target tasks. He was instructed to respond "yes" by pressing the thumbswitch held in the nonwriting hand, or "no" by pressing the thumbswitch held in the writing hand, as soon as he was certain of the correct response. The word target conditions required a correct answer to the question "Is the display word the same as (either one of) the target word(s)?" The letter target conditions required a correct answer to the question "Does the display word contain (either one of the target letter(s)?" Subjects knew that their responses and RTs were being recorded, but were given no knowledge of results during the experiment.

\section{Subjects}

The subjects were 32 volunteers from a subject pool at the University of Keele, aged from 18-28 years. Thirty subjects wrote with their right hand, and two with their left hand.

\section{RESULTS}

Although serial position of targets and letters in displays were fully balanced across conditions, no attempt was made to analyze serial position effects. Given the complexity of the determinants of positional gradients in letter-array perception, it seemed of little point to attempt to interpret such gradients in a design in which retinal position and spatial separation of letters was not varied, and in which the positioning of syllabic boundaries was not controlled. These features would be likely to impose a position effect on the data, quite irrespective of the ordering of "cognitive" operations on the stimuli (see, e.g., Spoehr \& Smith, 1973; Townsend, Taylor, \& Brown, 1971). Such confounding would make a serial position effect in the data virtually uninterpretable. Likewise, the left-right ordering of double targets when one of the targets was a match allowed no specific prediction about RT to be made. Even if subjects were to consistently compare the left-hand target with the stimulus before the other, one could not necessarily expect a match for the lefthand target to be faster. This would depend on added assumptions about the nature of the comparison task (e.g., whether it is exhaustive or self-terminating). Alternately, if there were a positional difference, it could well be explained by the subject's having a better representation of one or the other target, and would not argue for or against a particular kind of comparison process.
Table 2

Mean Reaction Time in Milliseconds

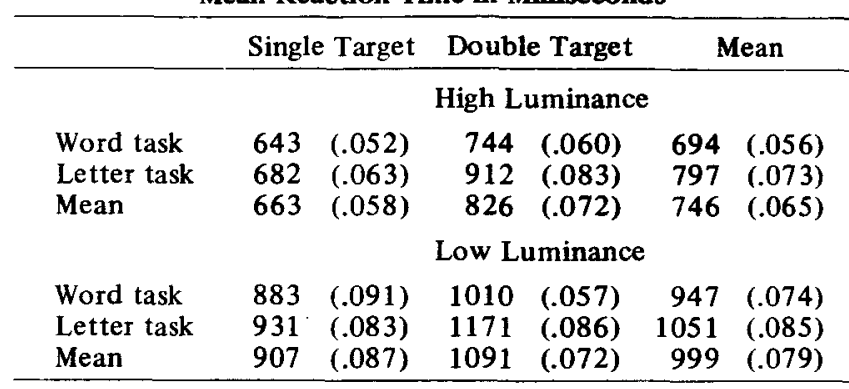

Note-Error proportions are given in parentheses.

Table 2 presents mean RTs to displays as a function of the three main factors of interest: task (word matching or letter matching), number of targets (one or two), and display luminance (high or low). An analysis of variance was carried out on the data for a four-within-subjects design (the additional factor being whether a "yes" or "no" response was required). Subjects and displays were treated as random effects and significance values were obtained by computing min $F^{\prime}$ ratios, as recommended by Clark (1973). All four main effects were significant. Mean word task RT was $817 \mathrm{msec}$, while mean letter task RT was $924 \mathrm{msec}$ $\left[\min F^{\prime}(1,41)=18.35, p<.001\right]$. This replicates once again the basic word-priority effect, with an RT advantage of $107 \mathrm{msec}$ for word matching over letter matching.

Mean RT for one target was $785 \mathrm{msec}$, while mean RT for two targets was $957 \mathrm{msec}$. This difference was highly significant $\left[\min F^{\prime}(1,41)=48.13, p<.001\right]$. Illumination level also had a highly significant effect upon RT $\left[\min \mathrm{F}^{\prime}(1,35)=41.41, \mathrm{p}<.001\right]$. The highluminance condition showed a mean RT of $746 \mathrm{msec}$, while the low-luminance condition showed a mean RT of $999 \mathrm{msec}$. Finally, "yes" responses were significantly faster than "no" responses [min $F^{\prime}(1,68)=8.42$, $\mathrm{p}<.01]$, with a mean "yes" RT of $848 \mathrm{msec}$, and a mean "no" RT of $893 \mathrm{msec}$.

Only one interaction was significant. This was the two-way interaction between task (word or letter match) and number of targets (one or two) $\left[\mathrm{min} \mathrm{F}^{\prime}(1,50)=8.72\right.$, $\mathrm{p}<.01]$. When there was one target, mean word task RT was $763 \mathrm{msec}$, while mean letter task RT was $806 \mathrm{msec}$, a word advantage of $43 \mathrm{msec}$. When there were two targets, mean word task RT was $877 \mathrm{msec}$, while mean letter task RT was $1,042 \mathrm{msec}$, a word advantage of $165 \mathrm{msec}$. Thus, increasing the number of iargets in the task from one to two increased the arithmetic RT difference between word and letter matching by $123 \mathrm{msec}$. On the other hand, the failure to find an interaction of Task by Luminance $\left[\min F^{\prime}(1,65)=.04\right]$ indicates that decreasing stimulus luminance did not significantly increase the arithmetic RT between word and letter matching; the mean RTs 
show this clearly. In the high-luminance condition, mean word task RT was $693 \mathrm{msec}$, while mean letter task RT was $797 \mathrm{msec}$, a word advantage of $104 \mathrm{msec}$. In the low-luminance condition, mean word task RT was $946 \mathrm{msec}$, while mean letter task RT was $1,051 \mathrm{msec}$, a word advantage of $105 \mathrm{msec}$.

In the preceding analysis, no account was taken of subjects' errors; that is to say, a subject's response was treated as correct in all cases. The dangers of ignoring errors have been cogently argued by Pachella (1974), on the grounds that speed-accuracy tradeoff may affect the RT data in important ways. Errors were therefore examined by subjecting error scores to an analysis of variance, carried out along identical lines to the RT analysis. Mean errors overall were $7.18 \%$. Of the main effects, only match vs mismatch was significant $\left[\min F^{\prime}(1,69)=13.01, p<.01\right]$. Subjects responded "no" in error on $9.95 \%$ of trials, but responded "yes" in error on only $4.40 \%$ of trials. Two interactions were also significant: Task by Match vs Mismatch $\left[\min F^{\prime}(1,58)=4.85, p<.05\right]$ and Number of Targets by Match vs Mismatch $\left[\min F^{\prime}(1,61)=4.26\right.$, $p<.05]$. When the target was a word, subjects responded "no" in error on 7.9\% of trials and "yes" on $5.1 \%$ of trials. When the target was a letter, subjects responded "no" in error on $12.0 \%$ of trials and "yes" on $3.7 \%$ of trials. Similarly, when there was one target, subjects responded "no" in error on $8.3 \%$ of trials and "yes" on $6.0 \%$ of trials. When there were two targets, subjects responded "no" in error on $11.5 \%$ of trials and "yes" on $3.0 \%$ of trials.

The error analysis shows that the significant error effects all occurred within experimental blocks, and never between them. In other words, overall error rates did not differ between conditions where the task, the number of targets, and the luminance of displays were manipulated, but only differed insofar as the distribution of "yes" vs "no" errors within these conditions was somewhat different. It seems justified, therefore, to treat RT differences as fairly reliable indicators of processing differences occurring as a result of manipulating the three main factors of interest.

\section{DISCUSSION}

The main RT effects are all consistent with the bulk of experimental literature in this field. The word-priority effect itself appears to be robust. Increases in RT with increases in size of memory set are well documented for many types of material (e.g., Juola \& Atkinson, 1971; Sternberg, 1969), and an RT advantage for matches over mismatches is a common finding, especially where mismatch stimuli are very similar to their targets (e.g., Juola, Fischler; Wood, \& Atkinson, 1971).

The failure to find any interactions between set size, luminance, and "yes" vs "no" responses is similar to the findings discussed by Sternberg (1969), and forms the basis of the conclusion that there are at least three independent stages within the information processing chain in such tasks. The interaction found of Task by Set Size, together with the lack of an interaction of Task by Luminance, indicates that the wordpriority effect is located at a stage whose duration is also affected by the size of the positive set. This stage is commonly called a "comparison" stage, and is to be distinguished from an "encoding" stage, influenced by such factors as luminance, but not, on the basis of these results, by whether the target is a word or a letter.

The error scores are consistent with the notion that subjects were adopting a rather cautious criterion in their detection of matches. The main effect of more "no" responses than "yes" responses is not in itself very informative, since it may equally well be due to response bias. A number of subjects reported that it seemed to them somehow more "natural" to respond with the writing hand, and a considerable number of the errors were described by subjects as "pressing the wrong button although I knew the right answer." However, response bias alone cannot account for the interactions which appeared, since the degree of bias should be the same for all conditions, given the ordering controls that were in operation. These interactions suggest that, as task difficulty increased (whether by decreasing luminance or by increasing positive set size), subjects became more cautious, and therefore more likely to respond "no" in error than to respond "yes" in error (more "misses" than "false positives").

If the three stages which this experiment has isolated may be called "encoding," "comparison," and "response selection," it still remains possible to ask whether there is some fourth stage, prior to the compairson stage, which may be producing a word-priority effect not manipulated in this experiment. Such a stage might perhaps be called "recognition." After all, there seems to be a difference between saying that words are encoded prior to their constituent letters and saying that they are recognized prior to their constituent letters. The former might be taken to be a statement about the way in which largely automatic pattern analysis programs run, while the latter might be taken to be a statement about the order in which results of various analyses appear in consciousness. It may then be argued that all this experiment has shown is that pattern analysis does not achieve results likely to lead to word recognition before it produces results likely to lead to letter recognition. It has not shown, however, that recognition uses results of encoding to produce conscious awareness of letters prior to, or even concurrently with, conscious awareness of words.

There are two counterarguments to this interpretation. The first argument rests on the slopes of the RT functions obtained in the Task by Number of Targets interaction. If these are extrapolated back to 
the $y$-intercept, there is no residual word-priority effect. Indeed there is a letter-priority effect. This extrapolation rests on two assumptions which need to be made explicit. First, that RT increases linearly with positive set size. Second, that subtracting the RT increase per single increase in set size from the RT for Set Size 1 gives a measure of the duration of the total process minus the comparison stage (i.e., the RT for a hypothetical Set Size 0 task). This subtractive operation gives a hypothetical word task RT of $649 \mathrm{msec}$, and a hypothetical letter task RT of $570 \mathrm{msec}$. There appears, therefore, to be no residual word-priority effect which another processing stage need account for. Of course, it is always possible to argue that a word-priority effect is present at another stage, at which it reduces what would have been a much larger letter-priority effect without it, but there is no empirical evidence to support any such view.

The second argument concerns the status of "recognition" as a "stage" in an information processing task. In brief, there seems no reason to suppose that within a particular task the objects of recognition are constant from trial to trial. To see that this is so, first take recognition to mean something similar to "the conscious awareness that a particular sensory experience has been identified or classified, together with the knowledge of the identification or classification given to it." On any such definition, it seems inappropriate to ask whether words are recognized prior to letters, for such a question presupposes that one is always consciously aware of words and letters in situations about which such questions are asked; it is not at all clear that this is the case. In the present experiment, for instance, it was clear that subjects did not always recognize words in the letter task. Immediately after the last experimental trial, some subjects were asked to name the word they had just seen. Several were unable to do this. Seemingly, they had recognized only the target letter (or its absence).

Any view of recognition as a process which results in conscious awareness is bound to render meaningless questions similar to "Are words recognized prior to letters?" However, perhaps recognition may be equated with some process not entailing consciousness, but separate from the stage affected by stimulus luminance which has been called encoding here. Specifically, one could suppose that the stage named encoding in the present paper might be something similar to the formation of an iconic image, in which lines, edges, and junctions are extracted from the stimulus information. There would then have to follow a stage at which the image would suffer subsequent transformations through contact with information in long-term memory, to provide letter and word codes. These two stages may be called Encoding 1 and Encoding 2. If this is a correct analysis, then the present experiment has shown that words are not processed faster than are letters at Encoding 1. A hypothesis which, therefore, remains a possibility is that words are processed faster than are letiers at Encoding 2. In order to test this hypothesis, it would be necessary to manipulate some experimental factor which is known to affect Encoding 2, but to affect neither the comparison stage nor Encoding 1. It is not obvious what such a factor might be; neither is it clear that the extrapolated letterpriority effect for target Set Size 0 could be plausibly located at any stage other than Encoding 2.

In summary, it has been demonstrated that a strong word-priority effect is located at a processing stage affected by manipulating target set size and commonly known as a comparison stage. It is suggested that the effect is determined by the number of comparisons to be made, which will always be greater for elemental targets, such as letters and phonemes, than for composite targets, such as words or syllables. The magnitude of the effect at the comparison stage seems sufficient to account for all the superiority of words over letters, without the invocation of a hypothesized encoding stage which favors word. Finally, it is argued that what is recognized (i.e., appears in consciousness) is not necessarily a clear indication of the way in which a stimulus is processed. In particular, the notion that recognition is a necessary prerequisite to correct response may not be helpful in such situations.

\section{REFERENCES}

Ball, F., Wood, C., \& Smith, E. E. When are semantic targets detected faster than visual or acoustic ones? Perception \& Psychophysics, 1975, 7, 1-8.

CLARK, H. H. The language-as-fixed-effect fallacy: A critique of language statistics in psychological research. Journal of Verbal Learning and Verbal Behavior, 1973, 12, 335-359.

Foss, D. J., \& Swinney, D. A. On the psychological reality of the phoneme. Journal of Verbal Learning and Verbal Behavior. 1973, 12, 246-257.

Johnson, N. F. On the function of letters in word identification: Some data and a preliminary model. Journal of Verbal Leaming and Verbal Behavior, 1975, 14, 17.29.

Juola, J. F., \& Atrinson, R. C. Memory scanning for words versus categories. Journal of Verbal Learning and Verbal Behavior, 1971, 10. 522-527.

Juola. J. F., Fischler, I., Wood, C. T., \& Atkinson, R. C. Recognition time for information in long-term memory. Perception \& Psychophysics, 1971, 10, 8-14.

MASSARo, D. W. Experimental psychology and information processing. Chicago: Rand McNally, 1975.

MCNEIL. D., \& Lindig, K. The perceptual reality of phonemes, syllables, words, and sentences. Journal of Verbal Learning and Verbal Behavior, 1973, 12, 419-430.

Pachella, R. G. The interpretation of reaction time in information-processing research. In B. H. Kantowitz (Ed.), Human information processing: Tutorials in performance and cognition. Potomac, Md: Lawrence Erlbaum, 1974.

Rejcher, G. M. Perceptual recognition as a function of meaningfulness of stimulus material. Journal of Experimental Psychology, 1969, 21, 275-280.

SAVIN, H. B., \& BEVER, T. G. The nonperceptual reality of the phoneme. Journal of Verbal Leaming and Verbal Behavior. 1970. 9, 295-302. 
Sloboda, J. A. Decision times for word and letter search: A holistic word identification model examined. Journal of Verbal Learning and Verbal Behavior, 1976, 15. 93-101.

SpoenR, K. T., \& Smith, E. E. The role of syllables in perceptual processing. Cognitive Psychology, 1973, 5, 71-89.

STERnBERG. S. The discovery of processing stages: Extensions of Donders' method. In W. G. Koster (Ed.), Attention and performance 1I, Amsterdam: North Holland, 1969.
Townsend, J. T., TAylor, S. G., \& Brown, D. R. Lateral masking for letters with unlimited viewing time. Perception \& Psychophysics, 1971, 10, 375-378.

WhEeler, D. D. Processes in word recognition. Cognitive Psychology, 1970, 1. 59-85.

(Received for publication July 9, 1976; revision accepted October $18,1976$. 